\title{
Outcomes in Patients with pT1-T2, pN0-N1 Breast Cancer After Conservative Surgery and Whole-breast Radiotherapy
}

\author{
MARIANNA TRIGNANI ${ }^{1}$, CLELIA DI CARLO ${ }^{1}$, CARMEN CEFALOGLI $^{1}$, MARIANNA NUZZO $^{1}$, \\ LUCIA ANNA URSINI $^{1}$, LUCIANA CARAVATTA $^{1}$, FRANCESCA PERROTTI ${ }^{1}$, \\ MARTA DI NICOLA ${ }^{2}$, AMBRA PAMIO $^{3}$ and DOMENICO GENOVESI ${ }^{1}$ \\ ${ }^{1}$ Department of Radiation Oncology, SS. Annunziata Hospital, ${ }^{2}$ Laboratory of Biostatistics, Department of Medical, \\ Oral and Biotechnological Sciences, ${ }^{3}$ Division of Hygene, Epidemiology and Public Health, \\ Department of Medicine and Science of Aging, G. D'Annunzio University of Chieti, Chieti, Italy
}

\begin{abstract}
Aim: To evaluate locoregional recurrence, overall survival, disease-free survival and prognostic influence of the number of positive lymph nodes and other variables in breast cancer treatment. Patients and Methods: A total of 377 patients with $p T 1-T 2, p N 0-N 1$ invasive breast carcinoma treated from 2005 to 2013 were retrospectively evaluated. Patients underwent conservative surgery followed by whole-breast radiotherapy. Clavicular region irradiation was not performed. Results: With a median follow-up of 4 years, locoregional recurrence rate was $3.4 \%$ (nodal recurrence $=1.06 \%$ ). Expression of progesterone receptors was significantly associated with better diseasefree survival, tumor size $(T>2 \mathrm{~cm})$ with poorer disease-free survival, locoregional recurrence and poorer overall survival; the presence of three nodal metastases was related to significantly poorer overall survival $(p=0.024)$. Conclusion: Whole-breast adjuvant radiotherapy without nodal irradiation after breast-conserving surgery led to low rate of locoregional recurrence and high rates of overall survival and disease-free survival in patients with pT1-T2 pNO-NI breast cancer.
\end{abstract}

Whole-breast adjuvant radiotherapy (WBRT) after conservative surgery represented a paradigmatic change in locoregional management of patients with breast cancer. Additional regional nodal irradiation in the case of four or

This article is freely accessible online.

Correspondence to: Domenico Genovesi, Department of Radiotherapy, "SS Annunziata" Hospital, Via Dei Vestini, 66100 Chieti, Italy. Tel: +39 08713558244, Fax: +39 08713557374, e-mail: d.genovesi@unich.it

Key Words: Breast cancer, pT1-T2, pN0-N1, adjuvant radiotherapy, conservative surgery. more positive axillary nodes reduces the risk of locoregional recurrence (LRR), improves overall survival (OS) and is recommended by current national and international guidelines. Conversely, there is no unique treatment at different radiotherapy centers for regional nodal irradiation in patients with $\mathrm{pN} 1$ breast cancer (1-4).

Data from Early Breast Cancer Trialists' Collaborative Group (EBCTCG) meta-analysis showed that WBRT reduces LRR and improves OS, both in patients with 1-3 positive lymph nodes and in the case of more than three positive lymph nodes (5); moreover, the National Cancer Institute of Canada Clinical Trial Group (MA.20 NCI-C) demonstrated that the nodal irradiation improves disease-free survival (DFS) $[82 \%$ vs. $77 \%$, hazard ratio $(\mathrm{HR})=0.86 ; p=0.01]$ without influencing OS $(82.8 \%$ vs. $81.8 \%, \mathrm{HR}=0.91$; $p=0.38$ ) (6). The fact is that the optimal treatment of regional nodes in the setting of breast-conserving surgery and radiation therapy is unclear (7-9).

Several authors attempted to define the classes of risk among patients with early-stage breast cancer and 1-3 positive axillary lymph nodes in relation to the expression of phenotypic characteristics of the tumor $(10,11)$. These and other experiences demonstrated that in such patients, nodal irradiation might be useful when meeting at least three of the following characteristics: estrogen (ER) and progesterone (PR) receptor negative, human epidermal growth factor receptor 2 (HER2neu) positive, young age ( $<50$ years), nodal ratio $\geq 25 \%$, presence of vascular invasion, medial tumor location, tumor diameter greater than $3 \mathrm{~cm}$ (12-17). Currently, this evidence suggests irradiation of the supraclavicular region, internal mammary and suspected axillary lymph nodes in patients with $\mathrm{N} 1$ disease. On the other hand, some authors believe it is unnecessary to prescribe nodal irradiation for patients with negative or one to three positive nodes due to the small number of isolated nodal relapses (clavicular recurrence rate $=0.4-0.9 \%$ in $\mathrm{N} 0,0.9-2.1 \%$ in $\mathrm{N} 1$ and $3.3-$ $5.5 \%$ in patients with $>5$ positive nodes) (7-14). 
The aim of our study was to determine long-term results (LRR, OS, DFS), prognostic significance of the number of positive lymph nodes and other prognostic variables such as HER2 and hormonal receptors status, grading, Ki67 and age, in patients with pT1-T2 pN0-N1 breast cancer who underwent conservative surgery followed by WBRT without clavicular irradiation.

\section{Patients and Methods}

A total of 377 female patients with pT1-T2 pN0-N1 breast cancer were treated at our Institute from December 2005 to December 2013 and retrospectively analyzed. In the case of $\mathrm{pN} 1$, according to our Institutional protocol, clavicular region irradiation was not performed. All patients were treated with external-beam WBRT using tangential fields technique with 6 or $15 \mathrm{MV}$ photons. The total delivered dose ranged between 42.5 and $54 \mathrm{~Gy}$ (2.6-2 Gy/fraction); 350 patients treated with conventional fractionation also received an electron boost to the tumor bed with total dose of $10 \mathrm{~Gy}$ ( $2 \mathrm{~Gy} /$ fraction).

Four cohorts were analyzed according to the number of involved axillary lymph nodes: N0 (no positive lymph nodes), $\mathrm{N} 1_{n 1}$ (one positive lymph node), $\mathrm{N} 1_{n 2}$ (two positive lymph nodes + ), $\mathrm{N} 1_{n 3}$ (three positive lymph nodes).

The characteristics of the patient population, medical history, pathological and immunohistochemical status and treatment details (chemo/hormone/radiation therapy) were collected for all patients and are summarized in Table I.

Statistical analysis. Continuous variables are reported as either mean and standard deviation (SD) or median and interquartile range (IQR) according to their distribution, as assessed by the Shapiro-Wilk test. Categorical variables are reported as absolute values and percentage.

For the survival analysis, the date of surgery was used as the start of observation. Survival time was calculated from the date of surgery to the date of last follow-up or the date of death. The date of followup was defined as the time interval between the date of the last clinic visit to our center (or the date of the last clinical correspondence) and the date of the surgery. LRR was defined as recurrence in the ipsilateral breast or in the axillary, supraclavicular, infra-clavicular or internal mammary lymph nodes. DFS was defined as the length of time before any evidence of LRR, distant metastasis or breast cancer-related death by the end of follow-up. Actuarial rates of total LRR, DFS and OS were calculated by the Kaplan-Meier method. Comparisons of clinical and pathological characteristics between patient groups were calculated using the log-rank test.

All statistical tests were evaluated at an alpha level of 0.05 . Statistical calculations were performed using the Statistical Package for Social Sciences (version 20.0; IBM Corp., Armonk, NY, USA).

\section{Results}

The median follow-up was 4 years (range $=2-10$ years); the database was updated to 2016, without any loss of patient.

The absence of lymph node involvement was found in 276 patients (73.2\%) while 1-3 pathological lymph nodes were reported in 101 patients.

The LRR was $3.4 \%$ (13/377), in particular 9/377 patients $(2.34 \%)$ presented breast recurrence and $4 / 377$ patients
$(1.06 \%)$ presented nodal recurrence: two within the axillary region, one in the supraclavicular region and one within the internal mammary chain. Of the four patients experiencing disease recurrence, three were $\mathrm{N}_{\mathrm{n} 1}$ and one $\mathrm{N}_{\mathrm{n} 3}$.

The rate of distant metastasis was $9 \%$ (34 patients out of 377 ); of these 34 patients, $82.3 \%$ have died (28 patients) due distant metastases, while six patients were still alive at the time of writing.

At the time of the analysis, 7.4\% (28/377) of patients had died due to breast disease-related causes, $5 \%$ to other causes, while $87.6 \%$ were still alive.

The OS for the whole cohort (pN0-N1) was $92.0 \pm 1.6 \%$ at 5 years and $78.7 \pm 3.7 \%$ at 8 years. By group, OS at 5 and 8 years was $92.3 \pm 1.9 \%$ and $80.6 \pm 4.1 \%$ for N0, $92.1 \pm 3.4 \%$ and $74.1 \pm 10.1 \%$ for $\mathrm{N} 1_{n 1}, 94.7 \pm 5.1 \%$ and $94.7 \% \pm 5.1 \%$ for $\mathrm{N} 1_{n 2}$, and $84.2 \pm 8.4 \%$ and $46.8 \pm 21.4 \%$ for $\mathrm{N}_{n 3}$. The presence of three lymph node metastases (19/377 patients of the sample) was significantly related to poorer OS ( $p$-value: $\mathrm{N}_{n 3}=0.024$, $\left.\mathrm{N} 1_{n 1}=0.175, \mathrm{~N} 1_{n 2}=0.369\right)$. In particular, out of $19 \mathrm{~N} 1_{n 3}$ patients, four died: one had distant metastases, and three died due to other non-oncological causes.

The DFS for the whole cohort (pN0-N1) at 5 and 8 years was $85.5 \pm 1.9 \%$ and $78.7 \pm 3.3 \%$, respectively. Specifically, at 5 and 8 years, DFS for those with N0 disease was $87.1 \pm 2.2 \%$ and $82.1 \pm 3.5 \%$, while in comparison it was $80.5 \pm 2.2 \%$ and $71.1 \pm 7.7 \%(p=0.034)$ for those with $\mathrm{N} 1_{n 1}, 89.5 \pm 7 \%$ and $89.5 \pm 7 \%(p=0.689)$ for those with $\mathrm{N}_{n 2}$, and $73.3 \pm 10.1 \%$ and $49.1 \pm 21.2 \%(p=0.031)$ for those with $\mathrm{N} 1_{n 3}$, respectively.

Univariate analysis for OS, DFS and LRR is shown in Table II. None of the examined variables such as ER, PR, menopausal status, adjuvant chemotherapy, HER2neu, margin status and grading, was significantly associated with OS or DFS. The previously mentioned variables did not seem to affect outcome of patients in our cohort, with the exception of PR in regard to LRR $(p=0.011)$.

Ki67 index (cut-off of reference $>14 \%$ ) was associated with an OS rate at 5 and 8 years of $81.4 \pm 3.2 \%$ and $73.4 \pm 5.4 \%(p=0.046)$ therefore, it did not appear to affect DFS nor LRR significantly.

Among the investigated variables, tumor size $>2 \mathrm{~cm}$ was significantly associated with analyzed outcomes: DFS was $87.3 \pm 3.7 \%$ at 5 years and $63.7 \pm 8.3 \%$ at 8 years; the LRR rate was $93.0 \pm 2.8 \%$ at both 5 and 8 years; OS was $76.3 \pm 4.6 \%$ and $64.3 \pm 7.6 \%$ at 5 and 8 years, respectively ( $p$-values of $0.002,0.042$ and 0.002 , respectively, for DFS, LRR and OS).

The Kaplan-Meier survival curves are shown in Figures 1 and 2 .

\section{Discussion}

WBRT after breast-conserving surgery associated with clavicular region irradiation represents the standard treatment 
Table I. Distribution of 377 patients with breast cancer according to selected clinicopathological features. Data are expressed as frequency (percentage).

\begin{tabular}{lccc}
\hline Characteristic & Number of patients $(\%)$ & Characteristic & Number of patients $(\%)$ \\
\hline All patients & 377 & Nodal status & $276(73.2)$ \\
Age (years) & & Negative & $63(16.7)$ \\
$\leq 40$ & $17(4.5)$ & 1 Positive & $19(5.0)$ \\
$41-50$ & $87(23.1)$ & 2 Positive & $19(5.0)$ \\
$51-59$ & $90(23.9)$ & 3 Positive & \\
$\geq 60$ & $183(48.5)$ & Margin status & $372(98.7)$ \\
Median (range) & $58.42(31-82)$ & Negative & $5(1.3)$ \\
Menopausal & & Positive & \\
Yes & $266(70.6)$ & ER status & \\
No & $111(29.4)$ & Negative & \\
Histology & $3(0.8)$ & Positive & $331(87.8)$ \\
pTis & $30(8.0)$ & PR status & \\
pT1a & Negative & $56(14.9)$ \\
pT1b & $17(19.1)$ & Positive & $321(85.1)$ \\
pT1c & Ki67 status & \\
pT2 & $93(24.7)$ & Negative & $229(60.7)$ \\
Adjuvant chemotherapy & Positive & $148(39.3)$ \\
Yes & $110(29.2)$ & HER2neu status & $337(89.4)$ \\
No & $267(70.8)$ & Negative & $40(10.6)$ \\
Grading & & Positive & $299(79.3)$ \\
G1 & $211(56.0)$ & Subtype & $31(8.2)$ \\
G2 & $133(35.3)$ & Luminal A & $35(9.3)$ \\
G3 & $33(8.8)$ & Luminal B & $12(3.2)$ \\
& & Triple-negative & HER2-overexpressing \\
\hline
\end{tabular}

ER: Estrogen receptor; PR: progesterone receptor; HER2neu: human epidermal growth factor receptor.

for patients with early tumor stage and four or more positive axillary lymph nodes (14).

Lacking randomized trials, the role of clavicular node irradiation is still debated in patients with early disease (pT1$\mathrm{T} 2)$ and few $(\leq 3)$ metastatic axillary lymph nodes, although certain studies suggest that in this group there are classes of high-risk patients who can benefit from radiotherapy to nodal stations in view of clinical and pathological risk factors: those with triple-negative disease, young age, nodal ratio $>25 \%$, with vascular invasion, extra-capsular extension, tumor size $>3 \mathrm{~cm}(15-17)$.

At our Radiotherapy Department, we performed wholebreast adjuvant radiotherapy without irradiation of clavicular nodes in patients with pT1-T2 pN0 pN1 breast cancer. The aim of this study was to evaluate LRR, OS, DFS and to determine the significance of prognostic variables, specially the number of positive lymph nodes, in the treatment of pT1T2 pN0-N1 breast cancer. Patients treated between 2006 and 2013 were retrospectively analyzed in order to avoid bias related to different therapeutic management prior to 2006. In our database pathological, immunohistochemistry data and information about chemotherapy and hormonal and radiation therapy were collected. Several studies have shown that some variables such as age, grading, receptor status, and chemotherapy are independent risk factors for LRR (18). In our study, no significant correlations were found between these variables and LRR, OS and DFS, with the exception of PR expression in regard to better LRR $(p=0.011)$.

In our series, tumor size $>2 \mathrm{~cm}$ was significantly correlated with poorer DFS, OS and LRR. The unclear role of tumor size was reported in literature showing that, although requiring the same therapeutic management, $\mathrm{T} 1$ and $\mathrm{T} 2$ stages cannot be considered equivalent, and they presumably require two different therapeutic approaches (18).

Ki67 is an emerging prognostic factor that seems to adversely influence outcome of patients with $\mathrm{N} 1$ disease; Ferguson and colleagues have shown that the proliferative index is one of the most important prognostic factors, but as yet it is not possible to tailor therapies according to the Ki67 value (18). In our study, the Ki67 index at the cut-off of $>14 \%$ did not appear to affect DFS nor LRR.

The prognostic role of the number of positive axillary lymph nodes is a hot topic investigated by several authors, but the lack of prospective and randomized studies has led to different conclusions. The meta-analysis of EBCTCG showed the benefit of WBRT on OS and reduction of 
in vivo $31: 151-158(2017)$

Table II. Univariate analysis of prognostic factors for overall survival (OS), locoregional recurrence (LRR) and disease-free survival (DFS). Data are expressed as the mean and standard error.

\begin{tabular}{|c|c|c|c|c|c|c|c|c|c|}
\hline \multirow[t]{2}{*}{ Factor } & \multicolumn{2}{|c|}{ OS $(\%)$} & \multirow[t]{2}{*}{$p$-Value } & \multicolumn{2}{|c|}{ LRR $(\%)$} & \multirow[t]{2}{*}{$p$-Value } & \multicolumn{2}{|c|}{ DFS (\%) } & \multirow[t]{2}{*}{$p$-Value } \\
\hline & 5 Years & 8 Years & & 5 Years & 8 Years & & 5 Years & 8 Years & \\
\hline Overall & $92.0 \pm 1.6$ & $78.7 \pm 3.7$ & & $96.7 \pm 0.9$ & $96.7 \pm 0.9$ & & $85.5 \pm 1.9$ & $78.7 \pm 3.3$ & \\
\hline \multicolumn{10}{|l|}{ Lymph nodes } \\
\hline Negative & $92.3 \pm 1.9$ & $80.6 \pm 4.1$ & & $97.7 \pm 0.9$ & $97.7 \pm 0.9$ & & $87.1 \pm 2.2$ & $82.1 \pm 3.5$ & \\
\hline 1 Positive & $92.1 \pm 3.4$ & $74.1 \pm 10.1$ & 0.175 & $91.9 \pm 3.5$ & $91.9 \pm 3.5$ & 0.020 & $80.5 \pm 5.1$ & $71.1 \pm 7.7$ & 0.034 \\
\hline 2 Positive & $94.7 \pm 5.1$ & $94.7 \pm 5.1$ & 0.369 & - & - & 0.509 & $89.5 \pm 7.0$ & $89.5 \pm 7.0$ & 0.689 \\
\hline 3 Positive & $84.2 \pm 8.4$ & $46.8 \pm 21.4$ & 0.024 & $94.4 \pm 5.4$ & $94.4 \pm 5.4$ & 0.383 & $73.7 \pm 10.1$ & $49.1 \pm 21.2$ & 0.031 \\
\hline \multicolumn{10}{|l|}{ ER } \\
\hline Negative & $91.3 \pm 4.2$ & $79.9 \pm 8.4$ & & - & - & & $80.0 \pm 6.0$ & $80.0 \pm 6.0$ & \\
\hline Positive & $92.1 \pm 1.7$ & $78.2 \pm 4.1$ & 0.992 & $96.2 \pm 1.1$ & $96.2 \pm 1.1$ & 0.189 & $86.2 \pm 2.0$ & $78.4 \pm 3.7$ & 0.465 \\
\hline \multicolumn{10}{|l|}{ PR } \\
\hline Positive & $93.1 \pm 1.6$ & $80.4 \pm 4.0$ & & $96.4 \pm 1.1$ & $96.4 \pm 1.1$ & 0.519 & $87.1 \pm 2.0$ & $79.3 \pm 3.7$ & 0.086 \\
\hline Negative & $85.7 \pm 4.7$ & $67.3 \pm 10.1$ & 0.011 & $98.2 \pm 1.8$ & $98.2 \pm 1.8$ & & $76.5 \pm 5.7$ & $76.5 \pm 5.7$ & \\
\hline \multicolumn{10}{|l|}{ HER2neu } \\
\hline Negative & $92.6 \pm 1.6$ & $79.1 \pm 3.9$ & & $96.9 \pm 1.0$ & $96.9 \pm 1.0$ & & $85.8 \pm 2.0$ & $79.9 \pm 3.3$ & \\
\hline Positive & $87.5 \pm 5.2$ & $76.6 \pm 11.2$ & 0.421 & $94.9 \pm 3.5$ & $94.9 \pm 3.5$ & 0.495 & $82.5 \pm 6.0$ & $70.7 \pm 12.1$ & 0.476 \\
\hline \multicolumn{10}{|l|}{ Ki67 } \\
\hline Negative & $91.3 \pm 2.2$ & $83.8 \pm 4.2$ & & $96.9 \pm 1.2$ & $96.9 \pm 1.2$ & & $87.9 \pm 2.4$ & $82.7 \pm 3.7$ & \\
\hline Positive & $92.8 \pm 2.2$ & $73.8 \pm 5.9$ & 0.263 & $96.4 \pm 1.6$ & $96.4 \pm 1.6$ & 0.891 & $81.4 \pm 3.2$ & $73.4 \pm 5.4$ & 0.046 \\
\hline \multicolumn{10}{|l|}{ Subtype } \\
\hline Luminal A & $92.4 \pm 1.8$ & $79.1 \pm 4.1$ & & $96.5 \pm 1.1$ & $96.5 \pm 1.1$ & & $86.2 \pm 2.1$ & $79.6 \pm 3.6$ & \\
\hline Luminal B & $86.9 \pm 6.1$ & $69.5 \pm 16.3$ & 0.203 & $93.4 \pm 4.5$ & $93.4 \pm 4.5$ & 0.381 & $83.7 \pm 6.7$ & $67.0 \pm 15.9$ & 0.489 \\
\hline Triple-negative & $97.1 \pm 2.8$ & $81.0 \pm 10.7$ & 0.490 & - & - & 0.270 & $85.0 \pm 6.2$ & $85.0 \pm 6.2$ & 0.910 \\
\hline HER2-overexpressing & $83.3 \pm 10.8$ & $83.3 \pm 10.8$ & 0.679 & - & - & 0.521 & $75.0 \pm 12.5$ & $75.0 \pm 12.5$ & 0.406 \\
\hline \multicolumn{10}{|l|}{ Menopausal } \\
\hline Yes & $91.4 \pm 2.0$ & $79.8 \pm 4.4$ & & $97.7 \pm 0.9$ & $97.7 \pm 0.9$ & & $87.5 \pm 2.2$ & $79.3 \pm 4.2$ & \\
\hline No & $93.6 \pm 2.3$ & $76.0 \pm 6.9$ & 0.710 & $94.1 \pm 2.4$ & $94.1 \pm 2.4$ & 0.104 & $80.4 \pm 3.9$ & $77.6 \pm 4.7$ & 0.114 \\
\hline \multicolumn{10}{|l|}{ Margin status } \\
\hline Negative & $91.9 \pm 1.6$ & $79.5 \pm 3.6$ & & $96.6 \pm 1.0$ & $96.6 \pm 1.0$ & & $85.2 \pm 1.9$ & $79.8 \pm 3.1$ & \\
\hline Positive & - & $75.0 \pm 21.7$ & 0.893 & - & - & & - & $750 \pm 21.7$ & 0.876 \\
\hline \multicolumn{10}{|l|}{ T-Stage } \\
\hline pT1 & $93.5 \pm 1.7$ & $85.1 \pm 3.5$ & & $97.8 \pm 0.9$ & $97.8 \pm 0.9$ & & $88.4 \pm 2.0$ & $84.5 \pm 3.0$ & \\
\hline pT2 & $87.3 \pm 3.7$ & $63.7 \pm 8.3$ & 0.002 & $93.0 \pm 2.8$ & $93.0 \pm 2.8$ & 0.042 & $76.3 \pm 4.6$ & $64.3 \pm 7.6$ & 0.002 \\
\hline \multicolumn{10}{|l|}{ Chemotherapy } \\
\hline Yes & $93.6 \pm 1.8$ & $77.4 \pm 6.0$ & & $95.3 \pm 2.1$ & $95.3 \pm 2.1$ & & $82.2 \pm 3.7$ & $75.7 \pm 5.0$ & \\
\hline No & $91.1 \pm 2.1$ & $78.9 \pm 4.9$ & 0.694 & $97.2 \pm 1.1$ & $97.2 \pm 1.1$ & 0.350 & $86.6 \pm 2.2$ & $79.2 \pm 4.6$ & 0.127 \\
\hline
\end{tabular}

ER: Estrogen receptor; PR: progesterone receptor; HER2neu: human epidermal growth factor receptor.

relapse, both in patients with 1-3 metastatic axillary nodes and in the case of more than three involved axillary lymph nodes (5). Data from the MA.20 NCI-C study showed that lymph node irradiation significantly affects DFS, but there was no difference in terms of LRR rate and OS (6). In 2007, Livi and colleagues published the results of a retrospective analysis of 4,185 patients with T1-T2 breast cancer treated with conservative surgery and whole-breast radiotherapy without nodal irradiation (14). In their series, only $1.7 \%$ of patients with one to three positive nodes experienced a nodal recurrence; no prognostic factor was found to be significant for nodal recurrence at the univariate analysis in this group, and having one to three positive nodes was not an independent prognostic factor at the multivariate analysis. The authors concluded that given the low rate of nodal recurrence, elective nodal irradiation for patients with $\mathrm{N} 1$ disease is not justified. However, the study was retrospective and developed over a long period of time, resulting in non-homogeneous management due to different treatment standards in different periods (4).

In 2010, a total of 5,717 patients with pT1-T4 breast cancer, treated at the University of Florence, were retrospectively analyzed by Livi et al. Patients were divided into three groups according to the number of positive axillary 

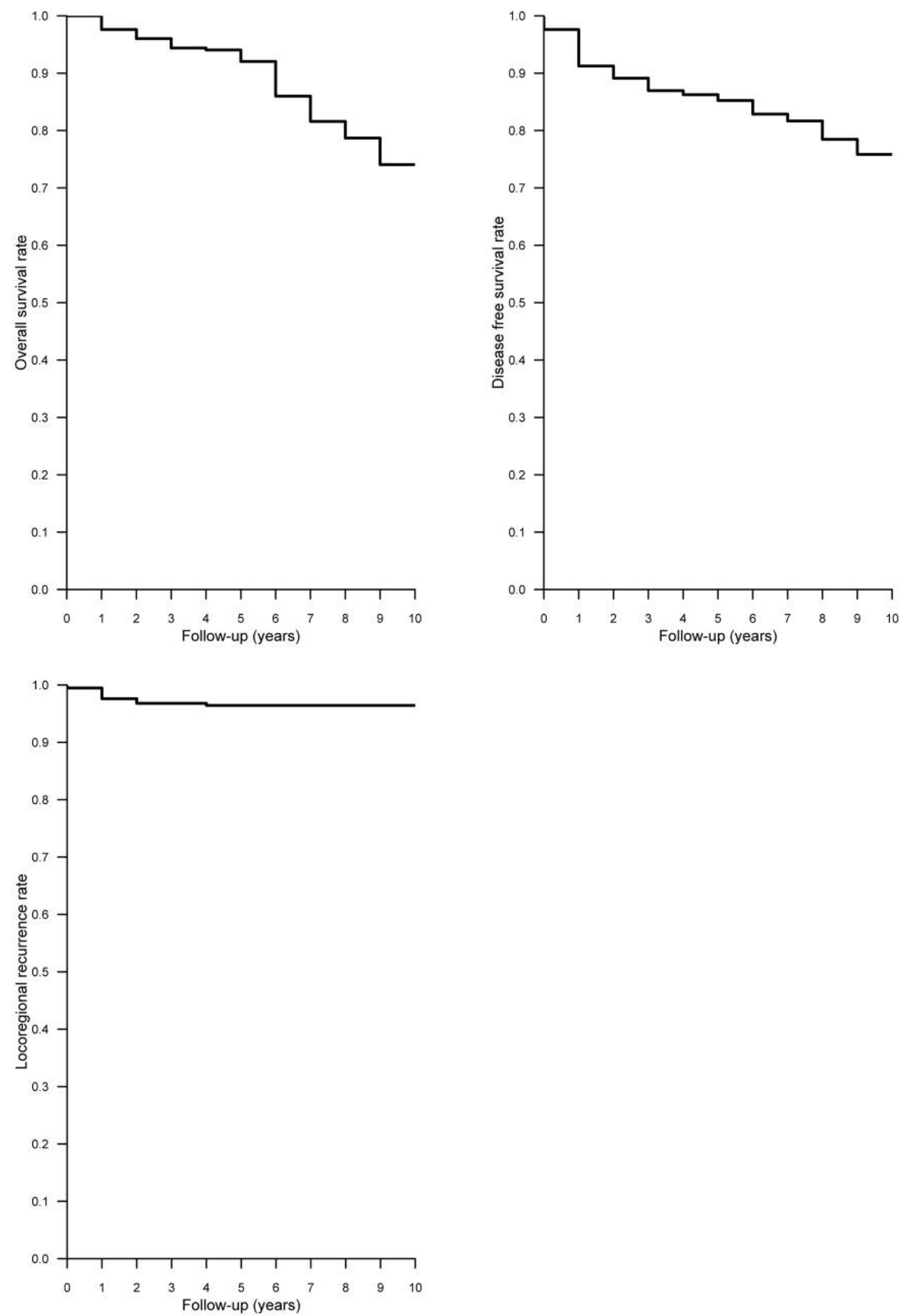

Figure 1. Kaplan-Meier curves of overall survival, disease-free survival and locoregional recurrence rate, for the whole cohort. 

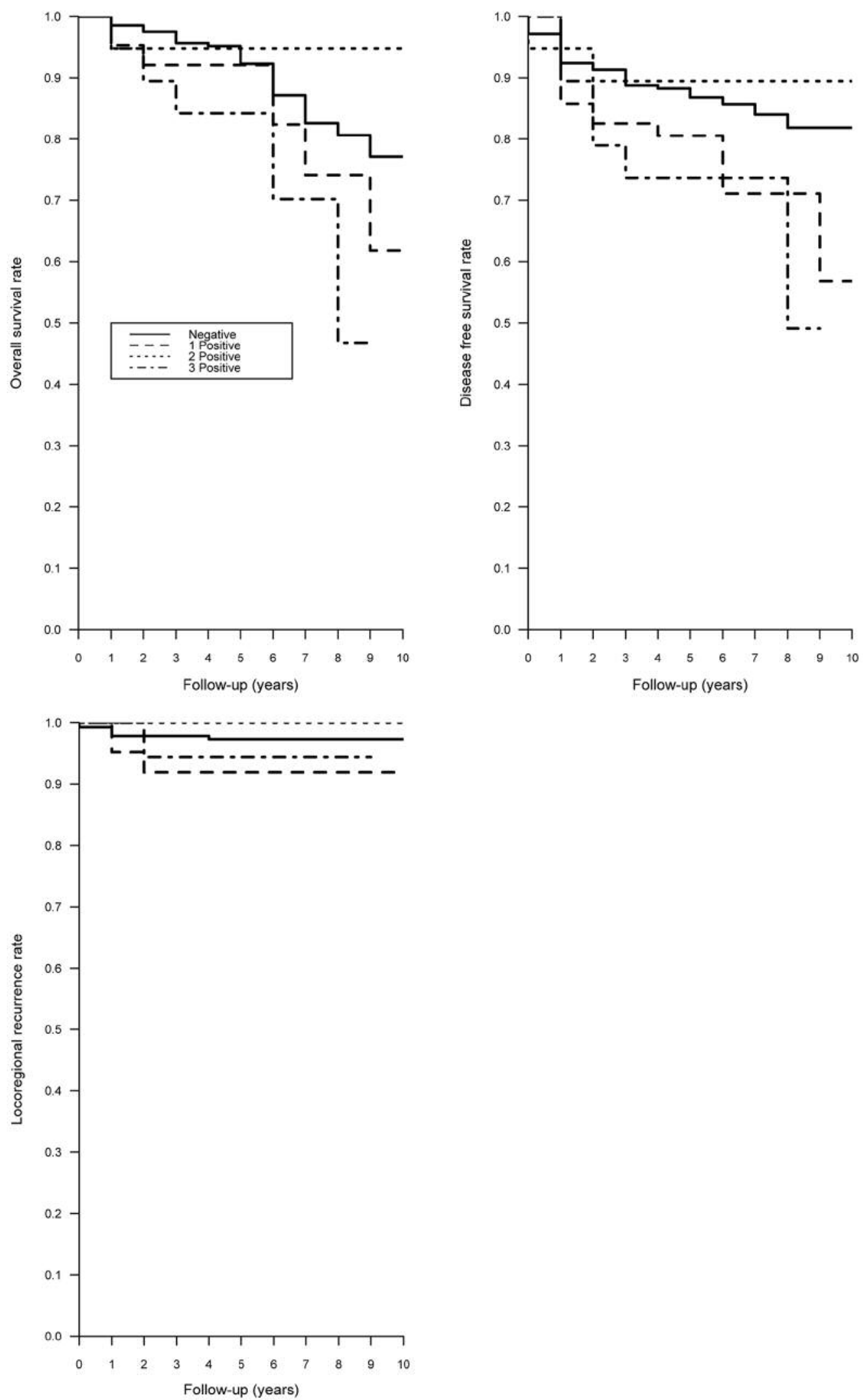

Figure 2. Kaplan-Meier curves of overall survival, disease-free survival and locoregional recurrence rate by lymph node positivity. 
nodes (PAN): P1, negative axillary lymph nodes; P2, one to three PAN; P3, >3 PAN (7). The authors recorded higher supraclavicular fossa recurrence in $\mathrm{P} 3$ patients compared to $\mathrm{P} 2$ and $\mathrm{P} 1$ patients.

In our study, the global LRR rate was $3.4 \%(13 / 377$ patients) and the nodal recurrence rate was $1.06 \%(4 / 377$ patients); the number of metastatic lymph nodes did not appear to have any significant impact on the risk of LRR, although in the subgroup analysis, the presence of one positive lymph node $\left(\mathrm{N} 1_{n 1}\right)$ significantly affected LRR $(p=0.02)$. The low number of relapses in our study and the small sub-sample size certainly contributed to this result.

Lu et al. retrospectively analyzed clinicopathological features and survival in 368 patients with pT1-T2 pN1 breast cancer presenting 1-3 histologically involved axillary lymph nodes, exploring prognosis to find high and low-risk groups (8). The 5- and 8-year recorded rates for the whole group were $7.2 \%$ and $10.7 \%$ for LRR, $85.1 \%$ and $77.7 \%$ for DFS, and $92.8 \%$ and $89.3 \%$ for OS. Similar results were reported in our study: for the whole group (pT1-T2 pN0-N1), the recorded rates at 5 and 8 years were $3.4 \pm 0.9 \%$ for $L R R$, $85.5 \pm 1.9 \%$ and $78.7 \pm 3.3 \%$ for DFS, $92.0 \pm 1.6 \%$ and $78.7 \pm 3.7$ for OS, respectively. In their study Lu et al. found that age $<40$ years, ER negativity, lymphovascular invasion and tumor size $>3 \mathrm{~cm}$ had a statistically significant negative influence on LRR and DFS; in our study a significant correlation was found only with tumor size $>2 \mathrm{~cm}$ in regard to poorer LRR, DFS and OS.

Regarding the impact of the number of positive axillary nodes on OS and DFS, in our study, at the univariate analysis, a significant negative association was found between N1n3 group and both OS and DFS.

Considering the small sample size, our results lead us to conclude, similarly to Livi et al., that patients with breast cancer presenting three or more positive lymph nodes could benefit from implementation of systemic therapies rather than nodal irradiation (7).

Our present study certainly suffers several limitations, such as the low number of events (recurrence/metastasis), small sub-sample size, and retrospective analysis. However, completeness of the database in terms of clinical, pathological and treatment information, homogeneity of management and update of follow-up until 2016, make our results reliable and allow the true relapse rate to be reported.

In our series, adjuvant radiotherapy after breastconserving surgery led to low rates of LRR and high rates of OS and DFS in patients with pT1-T2 pN0-N1 breast cancer. Moreover, despite its retrospective design and limited sample size, and given the low rate of nodal recurrence, the results from our study seem to suggest that nodal irradiation in patients presenting 1-3 positive axillary nodes is not necessary to improve outcome.

\section{Conflicts of Interest}

The Authors have stated that they have no conflict of interest in regard to this study.

\section{References}

1 Fisher B, Anderson S, Bryant J, Margolese RG, Deutsch M, Fisher ER, Jeong JH and Wolmark N: Twenty-year follow-up of a randomized trial comparing total mastectomy, lumpectomy plus irradiation for the treatment of invasive breast cancer. $\mathrm{N}$ Engl J Med 347: 1233-1241, 2002.

2 Can A: Breast-conserving therapy. Gland Surg 1: 84-86, 2012.

3 Truong PT, Jones SO, Kader HA, Wai ES, Speers CH, Alexander AS and Olivotto IA: Patients with T1 to T2 breast cancer with one to three positive nodes have higher local and regional recurrence risks compared with node negative patients after breast-conserving surgery and whole breast radiotherapy. Int $\mathbf{J}$ Radiat Oncol Biol Phys 73: 357-364, 2009.

4 Livi L, Paiar F, Saieva C, Scoccianti S, Dicosmo D, Borghesi S, Agresti B, Nosi F, Orzalesi L, Santini R, Barca R and Biti GP: Survival and breast relapse in 3834 patients with T1-T2 breast cancer after conserving surgery and adjuvant treatment. Radiother Oncol 82: 287-293, 2007.

5 Early Breast Cancer Trialists' Collaborative Group (EBCTCG): Effect of radiotherapy after breast-conserving surgery on 10-year recurrence and 15-year breast cancer death: meta-analysis of individual patient data for 10,801 women in 17 randomized trials. Lancet 378: 1707-1716, 2011.

6 Whelan TJ, Olivotto I, Parulekar WR, Ackerman I, Chua BH, Nabid A, Vallis KA, White JR, Rousseau P, Fortin A, Pierce LJ, Manchul L, Chafe S, Nolan MC, Craighead P, Bowen J, McCready DR, Pritchard KI, Gelmon K, Murray Y, Chapman JA, Chen BE and Levine MN: Regional nodal irradiation in early-stage breast cancer. N Engl J Med 373: 307-316, 2015.

7 Livi L, Scotti V, Saieva C, Meattini I, Detti B, Simontacchi G, Cardillo CD, Paiar F, Mangoni M, Marrazzo L, Agresti B, Cataliotti L, Bianchi S and Biti G: Outcome after conservative surgery and breast irradiation in 5,717 patients with breast cancer: implications for supraclavicular nodal irradiation. Int J Radiat Oncol Biol Phys 76: 978-983, 2010.

8 Lu C, Xu H, Chen X, Tong Z, Liu X and Jia Y: Irradiation after surgery for breast cancer patients with primary tumors and one to three positive axillary lymph nodes: Yes or no? Curr Oncol 20: e585-592, 2013.

9 Reddy SG and Kiel KD: Supraclavicular nodal failure in patients with one to three positive axillary lymph nodes treated with breast conserving surgery and breast irradiation, without supraclavicular node radiation. Breast J 13: 12-18, 2007.

10 Biancosino A, Bremer M, Karstens JH, Biancosino C and Meyer A: Postoperative periclavicular radiotherapy in breast cancer patients with 1-3 positive axillary lymph nodes. Outcome and morbidity. Strahlenther Onkol 188: 417-423, 2012.

11 Truong PT, Berthelet E, Lee J, Kader HA and Olivotto IA: The prognostic significance of the percentage of positive/dissected axillary lymph nodes in breast cancer recurrence and survival in patients with one to three positive axillary lymph nodes. Cancer 103: 2006-2014, 2005.

12 Truong PT, Olivotto IA, Kader HA, Panades M, Speers CH and Berthelet E: Selecting breast cancer patients with T1-T2 tumors 
and one to three positive axillary nodes at high postmastectomy locoregional recurrence risk for adjuvant radiotherapy. Int J Radiat Oncol Biol Phys 61: 1337-1347, 2005.

13 Poortmans PM, Collette S, Kirkove C, Van Limbergen E, Budach V, Struikmans H, Collette L, Fourquet A, Maingon P, Valli M, De Winter K, Marnitz S, Barillot I, Scandolaro L, Vonk E, Rodenhuis C, Marsiglia H, Weidner N, van Tienhoven G, Glanzmann C, Kuten A, Arriagada R, Bartelink H and Van den Bogaert W: Internal mammary and medial supraclavicular irradiation in breast cancer. N Engl J Med 373: 317-327, 2015.

14 Livi L, Barca R, Detti B, Fondelli S, Bastiani P, Santini R, Scotti V, Bianchi S, Cataliotti L, Mungai V and Biti G: Loco regional failure pattern after lumpectomy and breast irradiation in 4,185 patients with $\mathrm{T} 1$ and $\mathrm{T} 2$ breast cancer. Implications for nodal irradiation. Acta Oncol 45: 564-570, 2006.

15 Cheng SH, Tsai SY, Yu BL, Horng CF, Chen CM, Jian JJ, Chu NM, Tsou MH, Liu MC, Huang AT and Prosnitz LR: Validating a prognostic scoring system for postmastectomy locoregional recurrence in breast cancer. Int $\mathbf{J}$ Radiat Oncol Biol Phys 85: 953-958, 2013.
16 Wu SG, He ZY, Li FY, Wang JJ, Guo J, Lin Q and Guan XX: The clinical value of adjuvant radiotherapy in patients with early stage breast cancer with 1 to 3 positive lymph nodes after mastectomy. Chin J Cancer 29: 668-676, 2010.

17 Yang PS, Chen CM, Liu MC, Jian JM, Horng CF, Liu MJ, Yu BL, Lee MY and Chi CW: Radiotherapy can decrease locoregional recurrence and increase survival in mastectomy patients with $\mathrm{T} 1$ to $\mathrm{T} 2$ breast cancer and one to three positive nodes with negative estrogen receptor and positive lymphovascular invasion status. Int J Radiat Oncol Biol Phys 77: 516-522, 2010.

18 Ferguson NL, Bell J, Heidel R, Lee S, Vanmeter S, Duncan L, Munsey B, Panella T and Orucevic A: Prognostic value of breast cancer subtypes, Ki-67 proliferation index, age, and pathologic tumor characteristics on breast cancer survival in Caucasian women. Breast J 19: 22-30, 2013.

Received November 14, 2016

Revised December 9, 2016

Accepted December 21, 2016 